Article

\title{
Synthesis of spiropyrrolidine oxindoles through Rh(II)-catalyzed olefination/cyclization of diazooxindoles and vinyl azides
}

\author{
Ruxia Yi a,b,§, Leilei Qian a,b,§, Boshun Wan ${ }^{\mathrm{a}, *}$ \\ a Dalian Institute of Chemical Physics, Chinese Academy of Sciences, Dalian 116023, Liaoning, China \\ b University of Chinese Academy of Sciences, Beijing 100049, China
}

\section{A R T I C L E I N F O}

\section{Article history:}

Received 2 November 2018

Accepted 14 November 2018

Published 5 February 2019

\section{Keywords:}

Rhodium catalyst

Vinyl azides

Diazo compounds

Spiropyrrolidine oxindoles

Olefination

$[1+1+3]$ annulation

\begin{abstract}
A B S T R A C T
A simple and efficient process involving the $\mathrm{Rh}(\mathrm{II})$-catalyzed $[1+1+3]$ annulation of diazooxindoles and vinyl azides has been developed for the synthesis of spiropyrrolidine oxindoles with potential biological activity and significant synthetic applications. This process involves a novel rhodium-catalyzed olefination of diazo compounds, followed by annulation with vinyl azides. This method is compatible with a broad range of substrates and affords moderate to good yields under mild reaction conditions.
\end{abstract}

(C) 2019, Dalian Institute of Chemical Physics, Chinese Academy of Sciences. Published by Elsevier B.V. All rights reserved.

\section{Introduction}

Spirooxindoles, featuring a spiro ring fused at the 3-position of the oxindole core, have attracted considerable attention over the past decades owing to their unique structural properties and widespread occurrence in natural products [1-3]. In particular, molecules bearing a spiropyrrolidine oxindole skeleton often exhibit intriguing biological activities [4-6]. For example, as shown in Scheme 1, mitraphylline (compound I), a spirooxindole alkaloid isolated from the leaves of Mitragyna speciosa, shows antiproliferative effects, while compounds II and III exhibit antibacterial and antitumor activities, respectively [7,8]. In addition, these motifs find application in the synthesis of new ligands and catalysts [9]. In this regard, intense efforts have been devoted to their synthesis [2,10-12]. Thus far, most synthetic methods have focused on the 1,3-dipolar cycloaddi- tion of azomethine ylides [13-17] and the intramolecular cyclization of preformed precursors [18-24]. Despite these advances, there is still a high demand for the exploration of new catalytic routes for the bimolecular assembly of spiropyrrolidine oxindoles.

Vinyl azides, featuring both alkene and azide motifs, are versatile building blocks in the divergent synthesis of various azaheterocycles [25,26]. Moreover, diazo compounds have been widely employed as coupling partners in the annulation reaction. However, the cycloaddition of vinyl azides and diazo compounds has rarely been explored. Vinyl azides serve as two-atom partners in the reported Rh-catalyzed cyclopropanation [27] (Scheme 2a) and Cu-catalyzed [3+2] cycloaddition [28] (Scheme 2b) reactions. Following our previous studies on the azide chemistry [29-31] and cycloaddition reactions [32,33], we directed our attention towards developing new

\footnotetext{
* Corresponding author. Tel: +86-411-84379260; Fax: +86-411-84379223; E-mail: bswan@dicp.ac.cn

$\S$ Ruxia Yi and Leilei Qian contributed equally to this work.

This work was supported by the National Natural Science Foundation of China (21572225).

DOI: 10.1016/S1872-2067(18)63200-0 | http://www.sciencedirect.com/science/journal/18722067 | Chin. J. Catal., Vol. 40, No. 2, February 2019
} 


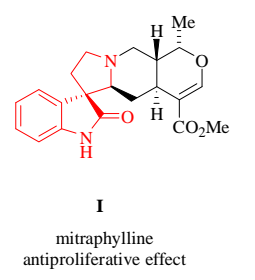

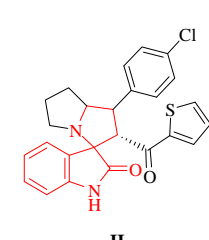

antibacterial effect

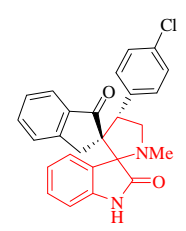

III

antitumor effect
Scheme 1. Bioactive spiropyrrolidine oxindoles.

a) Rh-catalyzed cyclopropanation

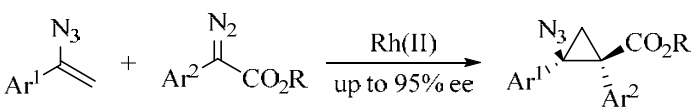

b) Cu-catalyzed cyclopentene synthesis

$$
\underbrace{N_{\text {EWG }}^{N_{2}}}_{E W G=\text { ester, COMe }} \stackrel{\mathrm{Cu}(\mathrm{I})}{\longrightarrow}
$$

c) Rh-catalyzed 1-pyrroline synthesis

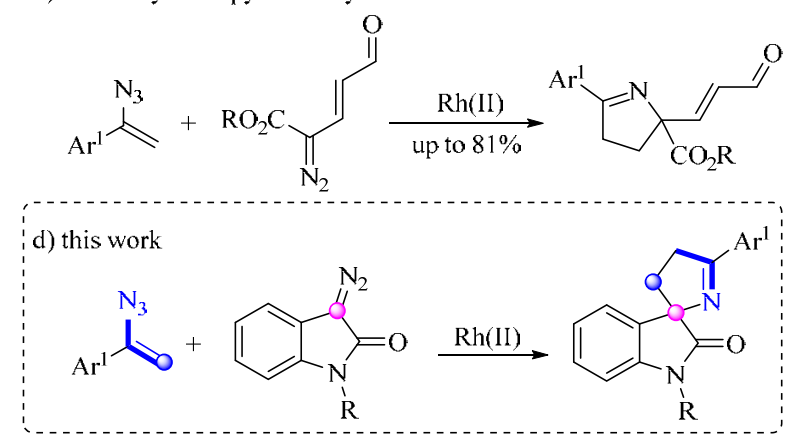

Scheme 2. Reactions of vinyl azides and diazo compounds.

cycloaddition reactions of vinyl azides and diazo compounds, in which vinyl azides may serve as three-atom synthons. Herein, we report a $\mathrm{Rh}(\mathrm{II})$-catalyzed [3+1+1] annulation of vinyl azides and 3-diazooxindoles, enabling the facile synthesis of spiropyrrolidine oxindoles (Scheme 2d). Coincidentally, while this work was underway, Katukojvala's group [34] reported the synthesis of functionalized 1-pyrrolines via a similar annulation of diazoenals and vinyl azides (Scheme 2c).

\section{Experimental}

\subsection{General information}

Commercially available reagents were used without further purification. Solvents were treated prior to use according to standard methods. All reactions were carried out under an argon atmosphere using standard Schlenk techniques or in an argon-filled glove box, unless otherwise noted. Column chromatography was carried out on silica gel (300-400 mesh) using a forced flow of eluent at a pressure of 0.3-0.5 bar. For thin-layer chromatography (TLC) experiments, silica gel GF254 was used and visualized by fluorescence quenching under UV light. NMR spectra were recorded on a Bruker $400 \mathrm{MHz}$ NMR spectrometer in the solvents indicated below. The ${ }^{1} \mathrm{H}$ and ${ }^{13} \mathrm{C}$ NMR chemical shifts were recorded in ppm downfield from the corresponding central peaks of $\mathrm{CDCl}_{3}$ (7.26 and $77.16 \mathrm{ppm}$, respectively), used as the internal standard. Coupling constants $(0)$ are reported in $\mathrm{Hz}$ and refer to apparent peak multiplications. The 3-diazooxindole and vinyl azide compounds were synthesized according to known literature procedures $[35,36]$.

\subsection{General procedure for the annulation of 3-diazooxindoles and vinyl azides}

Under argon atmosphere, dirhodium(II) tetra(trifluoroacetate) $\left(\mathrm{Rh}_{2}(\mathrm{TFA})_{4}, 2.5 \mathrm{~mol} \%\right)$ was added to a mixture of 3-diazooxindole $\mathbf{1}(0.3 \mathrm{mmol})$ and vinyl azide $\mathbf{2}$ (2.1 mmol) in 1,2-dichloroethane (DCE, $3 \mathrm{~mL}$ ). The mixture was stirred at $60{ }^{\circ} \mathrm{C}$ for $10 \mathrm{~h}$ until the substrate 1 was consumed. Then, the solvent was evaporated and the crude product was directly purified by flash column chromatography on silica gel (using petroleum ether/ethyl acetate as eluent) to give the desired product 3 .

\subsection{Product characterization data}

1-Methyl-5'-phenyl-3',4'-dihydrospiro[indoline-3,2'-pyrrol]2-one (3aa): yellow solid; $65.7 \mathrm{mg}$; 79\% yield; melting point (mp) $168-169{ }^{\circ} \mathrm{C} ;{ }^{1} \mathrm{H}$ NMR $\left(400 \mathrm{MHz}, \mathrm{CDCl}_{3}\right) \delta 7.92-7.90(\mathrm{~m}$, 2H), 7.48-7.38 (m, 3H), $7.32(\mathrm{td}, J=7.7,1.3 \mathrm{~Hz}, 1 \mathrm{H}), 7.14$ (dd, $J=$ $7.3,0.8 \mathrm{~Hz}, 1 \mathrm{H}), 7.06(\mathrm{td}, J=7.5,0.8 \mathrm{~Hz}, 1 \mathrm{H}), 6.87(\mathrm{~d}, J=7.8 \mathrm{~Hz}$, 1H), 3.49 (ddd, $J=16.7,9.6,6.9 \mathrm{~Hz}, 1 \mathrm{H}$ ), 3.37 (ddd, $J=17.0,9.7$, $5.2 \mathrm{~Hz}, 1 \mathrm{H}$ ), 3.24 (s, 3H), 2.62 (ddd, $J=13.2,9.6,5.2 \mathrm{~Hz}, 1 \mathrm{H}$ ), 2.29 (ddd, $J=13.2,9.7,6.9 \mathrm{~Hz}, 1 \mathrm{H}) ;{ }^{13} \mathrm{C} \mathrm{NMR}\left(100 \mathrm{MHz} \mathrm{CDCl}_{3}\right) \delta$ $177.4,177.3,143.8,133.9,132.2,131.2,129.3,128.5,128.4$, 123.7, 123.1, 108.4, 81.6, 36.9, 32.8, 26.5; high-resolution mass spectrometry (HRMS, Q-TOF, ESI) calcd for $\mathrm{C}_{18} \mathrm{H}_{17} \mathrm{~N}_{2} \mathrm{O}^{+}[\mathrm{M}+\mathrm{H}]^{+}$ 277.1335, found 277.1339.

1-Benzyl-5'-phenyl-3',4'-dihydrospiro[indoline-3,2'-pyrrol]2-one (3ba): yellow solid; $93.0 \mathrm{mg}$; 88\% yield; mp $137-138{ }^{\circ} \mathrm{C}$; ${ }^{1} \mathrm{H}$ NMR (400 MHz, $\left.\mathrm{CDCl}_{3}\right) \delta$ 7.94-7.91 (m, 2H), 7.47-7.40 (m, $3 \mathrm{H}), 7.35-7.29(\mathrm{~m}, 4 \mathrm{H}), 7.27-7.23(\mathrm{~m}, 1 \mathrm{H}), 7.19-7.13(\mathrm{~m}, 2 \mathrm{H})$, 7.02-6.98 (dd, $J=11.0,4.0 \mathrm{~Hz}, 1 \mathrm{H}), 6.74(\mathrm{~d}, J=7.8 \mathrm{~Hz}, 1 \mathrm{H}), 5.02$ (d, $J=15.7 \mathrm{~Hz}, 1 \mathrm{H}), 4.83$ (d, $J=15.7 \mathrm{~Hz}, 1 \mathrm{H}$ ), 3.50 (ddd, $J=16.8$, 9.5, 7.2 Hz, 1H), 3.38 (ddd, $J=17.0,9.7,4.9 \mathrm{~Hz}, 1 \mathrm{H}$ ), 2.67 (ddd, $J$ $=13.4,9.5,4.9 \mathrm{~Hz}, 1 \mathrm{H}$ ), 2.31 (ddd, $J=13.1,9.7,7.2 \mathrm{~Hz}, 1 \mathrm{H}$ ); ${ }^{13} \mathrm{C}$ NMR $\left(100 \mathrm{MHz}, \mathrm{CDCl}_{3}\right) \delta 177.57,177.43,142.87,135.80$, 133.86, 132.31, 131.22, 129.16, 128.87, 128.50, 128.40, 127.67, 127.41, 123.75, 123.17, 109.42, 81.62, 44.04, 36.84, 33.22; HRMS (Q-TOF, ESI) calcd for $\mathrm{C}_{24} \mathrm{H}_{21} \mathrm{~N}_{2} \mathrm{O}^{+}[\mathrm{M}+\mathrm{H}]^{+} 353.1648$, found 353.1649 .

1,5'-Diphenyl-3',4'-dihydrospiro[indoline-3,2'-pyrrol]-2-one (3ca): yellow solid; $90.0 \mathrm{mg}$; 89\% yield; mp $121-122{ }^{\circ} \mathrm{C} ;{ }^{1} \mathrm{H}$ NMR (400 MHz, $\left.\mathrm{CDCl}_{3}\right) \delta$ 7.94-7.92 (m, 2H), 7.52-7.36 (m, 8H), 7.25-7.19 (m, 2H), 7.07 (t, $J=7.2 \mathrm{~Hz}, 1 \mathrm{H}), 6.88(\mathrm{~d}, J=7.9 \mathrm{~Hz}$, 1H), 3.52-3.39 (m, 2H), 2.73 (ddd, $J=14.4,9.5,5.0 \mathrm{~Hz}, 1 \mathrm{H}$ ), 2.37 (ddd, $J=13.1,9.6,7.2 \mathrm{~Hz}, 1 \mathrm{H}) ;{ }^{13} \mathrm{C}$ NMR $\left(100 \mathrm{MHz}, \mathrm{CDCl}_{3}\right) \delta$ 177.7, 176.6, 143.6, 134.5, 133.8, 132.1, 131.2, 129.6, 129.1, 128.5, 128.4, 128.0, 126.5, 124.0, 123.6, 109.7, 81.7, 36.9, 33.6; HRMS (Q-TOF, ESI) calcd for $\mathrm{C}_{23} \mathrm{H}_{19} \mathrm{~N}_{2} \mathrm{O}^{+}[\mathrm{M}+\mathrm{H}]^{+} 339.1492$, 
found 339.1493.

1-Benzoyl-5'-phenyl-3',4'-dihydrospiro[indoline-3,2'-pyrrol ]-2-one (3da): yellow solid; $100.5 \mathrm{mg}$; $91 \%$ yield; mp 129-130 ${ }^{\circ} \mathrm{C}$; ${ }^{1} \mathrm{H}$ NMR (400 MHz, $\left.\mathrm{CDCl}_{3}\right) \delta$ 7.95-7.89 (m, 3H), 7.77-7.75 (m, 2H), 7.57-7.53 (m, 1H), 7.49-7.39 (m, 6H), $7.24(\mathrm{~d}, J=3.7$ $\mathrm{Hz}, 2 \mathrm{H}$ ), 3.47-3.33 (m, 2H), 2.67 (ddd, $J=13.7,8.4,5.4 \mathrm{~Hz}, 1 \mathrm{H}$ ), 2.38-2.30 (m, 1H); ${ }^{13} \mathrm{C}$ NMR (100 MHz, $\left.\mathrm{CDCl}_{3}\right) \delta 178.0,177.0$, $169.5,140.2,134.2,133.6,132.9,131.8,131.5,129.7,129.5$, 128.6, 128.5, 128.3, 125.7, 123.9, 115.5, 82.1, 37.0, 33.9; HRMS (Q-TOF, ESI) calcd for $\mathrm{C}_{24} \mathrm{H}_{19} \mathrm{~N}_{2} \mathrm{O}_{2}{ }^{+}[\mathrm{M}+\mathrm{H}]+367.1441$, found 367.1444

5'-Phenyl-1-tosyl-3',4'-dihydrospiro[indoline-3,2'-pyrrol]-2one (3ea): yellow solid; $112.3 \mathrm{mg} ; 90 \%$ yield; mp $70-71{ }^{\circ} \mathrm{C} ;{ }^{1} \mathrm{H}$ NMR (400 MHz, $\left.\mathrm{CDCl}_{3}\right) \delta 8.00(\mathrm{~d}, J=8.4 \mathrm{~Hz}, 2 \mathrm{H}), 7.95(\mathrm{~d}, J=8.2$ $\mathrm{Hz}, 1 \mathrm{H}), 7.84-7.82(\mathrm{~m}, 2 \mathrm{H}), 7.48-7.45(\mathrm{~m}, 1 \mathrm{H}), 7.41-7.36(\mathrm{~m}$, $3 \mathrm{H}), 7.31(\mathrm{~d}, J=8.1 \mathrm{~Hz}, 2 \mathrm{H}), 7.20-7.12(\mathrm{~m}, 2 \mathrm{H}), 3.44-3.29(\mathrm{~m}$, $2 \mathrm{H}$ ), 2.61 (ddd, $J=13.4,8.8,4.7 \mathrm{~Hz}, 1 \mathrm{H}$ ), 2.41 (s, 3H), 2.21 (ddd, $J=13.4,9.4,8.3 \mathrm{~Hz}, 1 \mathrm{H}) ;{ }^{13} \mathrm{C} \mathrm{NMR}\left(100 \mathrm{MHz}, \mathrm{CDCl}_{3}\right) \delta 178.8$, 175.4, 145.8, 139.0, 135.3, 133.4, 131.6, 131.2, 130.0, 129.9, 128.6, 128.5, 128.1, 125.5, 124.2, 113.9, 81.6, 36.6, 34.7, 21.8; HRMS (Q-TOF, ESI) calcd for $\mathrm{C}_{24} \mathrm{H}_{21} \mathrm{~N}_{2} \mathrm{O}_{3} \mathrm{~S}^{+}[\mathrm{M}+\mathrm{H}]^{+}$417.1267, found 417.1266.

1-(Methylsulfonyl)-5'-phenyl-3',4'-dihydrospiro[indoline-3, 2'-pyrrol]-2-one (3fa): yellow solid; $76.0 \mathrm{mg} ; 74 \%$ yield; $\mathrm{mp}$ 163-164 ${ }^{\circ} \mathrm{C}$; ${ }^{1} \mathrm{H}$ NMR $\left(400 \mathrm{MHz}, \mathrm{CDCl}_{3}\right) \delta 7.91-7.89(\mathrm{~m}, 2 \mathrm{H})$, $7.81(\mathrm{~d}, J=8.2 \mathrm{~Hz}, 1 \mathrm{H}), 7.52-7.41(\mathrm{dt}, J=25.9,7.2 \mathrm{~Hz}, 3 \mathrm{H})$, 7.38-7.34 (m, 1H), 7.23-7.17 (m, 2H), 3.51-3.36 (m, 5H), 2.72 (ddd, $J=13.8,9.0,5.0 \mathrm{~Hz}, 1 \mathrm{H}$ ), 2.30 (ddd, $J=13.4,9.4,7.8 \mathrm{~Hz}$, 1H); $\left.{ }^{13} \mathrm{C} \mathrm{NMR} \mathrm{(100} \mathrm{MHz,} \mathrm{CDCl}_{3}\right) \delta 178.8,176.5,138.8,133.4$, 131.7, 131.1, 130.0, 128.7, 128.5, 125.6, 124.3, 113.8, 81.7, 41.8, 36.8, 34.4; HRMS (Q-TOF, ESI) calcd for $\mathrm{C}_{18} \mathrm{H}_{17} \mathrm{~N}_{2} \mathrm{O}_{3} \mathrm{~S}^{+}[\mathrm{M}+\mathrm{H}]^{+}$ 341.0954, found 341.0960.

5'-Phenyl-3',4'-dihydrospiro[indoline-3,2'-pyrrol]-2-one (3ga): yellow solid; $61.8 \mathrm{mg} ; 79 \%$ yield; mp $217-218{ }^{\circ} \mathrm{C} ;{ }^{1} \mathrm{H}$ NMR (400 MHz, $\left.\mathrm{CDCl}_{3}\right) \delta 9.12(\mathrm{~s}, 1 \mathrm{H}), 7.94(\mathrm{~d}, J=7.1 \mathrm{~Hz}, 2 \mathrm{H})$, $7.49-7.40(\mathrm{~m}, 3 \mathrm{H}), 7.19$ (t, $J=7.6 \mathrm{~Hz}, 1 \mathrm{H}), 7.11(\mathrm{~d}, J=7.3 \mathrm{~Hz}$, 1H), 7.00 (t, $J=7.5 \mathrm{~Hz}, 1 \mathrm{H}$ ), $6.91(\mathrm{~d}, J=7.7 \mathrm{~Hz}, 1 \mathrm{H}$ ), 3.49 (ddd, $J=$ 16.6, 9.4, 7.0 Hz, 1H), 3.42-3.34 (m, 1H), 2.65 (ddd, $J=14.5,9.6$, $5.3 \mathrm{~Hz}, 1 \mathrm{H}), 2.30$ (ddd, $J=13.2,9.6,7.0 \mathrm{~Hz}, 1 \mathrm{H}) ;{ }^{13} \mathrm{C}$ NMR $(100$ $\mathrm{MHz}_{\mathrm{CDCl}}$ ) $\delta 180.0,177.6,141.0,133.8,132.8,131.3,129.3$, 128.6, 128.4, 123.9, 123.0, 110.4, 82.1, 36.8, 33.0; HRMS (Q-TOF, ESI) calcd for $\mathrm{C}_{17} \mathrm{H}_{15} \mathrm{~N}_{2} \mathrm{O}^{+}[\mathrm{M}+\mathrm{H}]+263.1179$, found 263.1180 .

1-Benzoyl-6-chloro-5'-phenyl-3',4'-dihydrospiro[indoline-3, 2'-pyrrol]-2-one (3ha): yellow solid; $99.6 \mathrm{mg}$; 83\% yield; $\mathrm{mp}$ $119-120^{\circ} \mathrm{C} ;{ }^{1} \mathrm{H}$ NMR $\left(400 \mathrm{MHz}, \mathrm{CDCl}_{3}\right) \delta 8.03(\mathrm{~d}, J=1.7 \mathrm{~Hz}, 1 \mathrm{H})$, $7.92-7.90(\mathrm{~m}, 2 \mathrm{H}), 7.78-7.76(\mathrm{~m}, 2 \mathrm{H}), 7.58(\mathrm{t}, J=7.5 \mathrm{~Hz}, 1 \mathrm{H})$, 7.52-7.42 (m, 5H), 7.25-7.17 (m, 2H), 3.45-3.38 (m, 2H), 2.68 (ddd, $J=13.6,8.5,5.3 \mathrm{~Hz}, 1 \mathrm{H}), 2.36-2.25(\mathrm{~m}, 1 \mathrm{H}) ;{ }^{13} \mathrm{C}$ NMR $(100$ $\mathrm{MHz}_{\mathrm{CDCl}}$ ) $\delta 178.3,176.6,169.2,141.0,135.4,133.8,133.4$, $133.1,131.7,130.2,129.5,128.7,128.5,128.3,125.7,124.9$, 116.1, 81.8, 37.0, 33.7; HRMS (Q-TOF, ESI) calcd for $\mathrm{C}_{24} \mathrm{H}_{18} \mathrm{ClN}_{2} \mathrm{O}_{2}+[\mathrm{M}+\mathrm{H}]+401.1051$, found 401.1057 .

1-Benzoyl-7-chloro-5'-phenyl-3',4'-dihydrospiro[indoline-3, 2'-pyrrol]-2-one (3ia): yellow solid; $90.0 \mathrm{mg}$; 75\% yield; $\mathrm{mp}$ 191-192 ${ }^{\circ} \mathrm{C}$; ${ }^{1} \mathrm{H}$ NMR $\left(400 \mathrm{MHz}, \mathrm{CDCl}_{3}\right) \delta 8.08-8.06(\mathrm{~m}, 2 \mathrm{H})$, 7.92-7.90 (m, 2H), $7.64(\mathrm{t}, J=7.4 \mathrm{~Hz}, 1 \mathrm{H}), 7.53-7.36(\mathrm{~m}, 6 \mathrm{H})$,
7.20-7.14 (m, 2H), 3.48-3.34 (m, 2H), 2.71 (ddd, $J=13.6,8.6$, $5.1 \mathrm{~Hz}, 1 \mathrm{H}), 2.40-2.32(\mathrm{~m}, 1 \mathrm{H}) ;{ }^{13} \mathrm{C}$ NMR $\left(100 \mathrm{MHz}, \mathrm{CDCl}_{3}\right) \delta$ 178.5, 177.1, 168.6, 138.3, 135.1, 134.5, 133.4, 133.2, 131.6, 131.1, 128.7, 128.6, 128.4, 126.2, 122.3, 119.8, 82.9, 36.9, 34.2; HRMS (Q-TOF, ESI) calcd for $\mathrm{C}_{24} \mathrm{H}_{18} \mathrm{ClN}_{2} \mathrm{O}_{2}+[\mathrm{M}+\mathrm{H}]^{+} 401.1051$, found 401.1056.

1-Benzoyl-5-chloro-5'-phenyl-3',4'-dihydrospiro[indoline-3, 2'-pyrrol]-2-one (3ja): yellow solid; $102.8 \mathrm{mg}$; 85\% yield; mp $143-144{ }^{\circ} \mathrm{C}$; ${ }^{1} \mathrm{H}$ NMR $\left(400 \mathrm{MHz}, \mathrm{CDCl}_{3}\right) \delta 7.94-7.90(\mathrm{~m}, 3 \mathrm{H})$, 7.77-7.75 (m, 2H), $7.57(\mathrm{t}, J=7.3 \mathrm{~Hz}, 1 \mathrm{H}), 7.52-7.39(\mathrm{~m}, 6 \mathrm{H})$, 7.23-7.25 (m, 1H), 3.44-3.40 (m, 2H), 2.69 (ddd, $J=13.5,8.1$, $5.7 \mathrm{~Hz}, 1 \mathrm{H}), 2.37-2.29(\mathrm{~m}, 1 \mathrm{H}) ;{ }^{13} \mathrm{C}$ NMR $\left(100 \mathrm{MHz}, \mathrm{CDCl}_{3}\right) \delta$ 178.6, 176.3, 169.2, 138.7, 133.9, 133.5, 133.3, 133.0, 131.7, 131.1, 129.7, 129.5, 128.7, 128.5, 128.3, 124.3, 116.9, 81.9, 37.0, 33.8; HRMS (Q-TOF, ESI) calcd for $\mathrm{C}_{24} \mathrm{H}_{18} \mathrm{ClN}_{2} \mathrm{O}_{2}+[\mathrm{M}+\mathrm{H}]^{+}$ 401.1051, found 401.1060.

1-Benzoyl-5-fluoro-5'-phenyl-3',4'-dihydrospiro[indoline-3, 2'-pyrrol]-2-one (3ka): yellow solid; $94.0 \mathrm{mg}$; 82\% yield; $\mathrm{mp}$ 193-194 ${ }^{\circ} \mathrm{C}$; ${ }^{1} \mathrm{H}$ NMR (400 MHz, $\left.\mathrm{CDCl}_{3}\right) \delta 7.97$ (dd, $J=8.9,4.5$ $\mathrm{Hz}, 1 \mathrm{H}), 7.92-7.90(\mathrm{~m}, 2 \mathrm{H}), 7.77-7.74(\mathrm{~m}, 2 \mathrm{H}), 7.57(\mathrm{t}, J=7.5$ $\mathrm{Hz}, 1 \mathrm{H}), 7.52-7.42$ (m, 5H), 7.13 (td, $J=9.0,2.7 \mathrm{~Hz}, 1 \mathrm{H}), 6.98$ (dd, $J=7.5,2.7 \mathrm{~Hz}, 1 \mathrm{H}$ ), 3.44-3.39 (m, 2H), 2.69 (ddd, $J=13.6$, 8.6, $5.1 \mathrm{~Hz}, 1 \mathrm{H}), 2.36-2.28(\mathrm{~m}, 1 \mathrm{H}) ;{ }^{13} \mathrm{C}$ NMR $\left(100 \mathrm{MHz}, \mathrm{CDCl}_{3}\right) \delta$ 178.6, 176.6, 169.3, 160.7 (d, $J=244.9 \mathrm{~Hz}), 136.1$ (d, $J=2.5 \mathrm{~Hz}$ ), 134.1, 133.6 (d, $J=7.9 \mathrm{~Hz}), 133.3,132.9,131.7,129.4,128.7$, 128.5, 128.3, 117.1 (d, $J=7.9 \mathrm{~Hz}), 116.3$ (d, $J=23.0 \mathrm{~Hz}), 111.4$ (d, $J=24.3 \mathrm{~Hz}), 82.0,37.0,33.9 ;{ }^{19} \mathrm{~F}$ NMR $\left(376 \mathrm{MHz} \mathrm{CDCl}_{3}\right) \delta$ -115.99; HRMS (Q-TOF, ESI) calcd for $\mathrm{C}_{24} \mathrm{H}_{18} \mathrm{FN}_{2} \mathrm{O}_{2}{ }^{+}[\mathrm{M}+\mathrm{H}]^{+}$ 385.1347, found 385.1331.

1-Benzoyl-5-bromo-5'-phenyl-3',4'-dihydrospiro[indoline-3, 2'-pyrrol]-2-one (3la): yellow solid; $119.0 \mathrm{mg}$; $89 \%$ yield; mp $141-142{ }^{\circ} \mathrm{C}$; ${ }^{1} \mathrm{H}$ NMR $\left(400 \mathrm{MHz}, \mathrm{CDCl}_{3}\right) \delta$ 7.94-7.85 (m, 3H), 7.76-7.74 (m, 2H), 7.60-7.37 (m, 7H), 7.37 (d, $J=2.0 \mathrm{~Hz}, 1 \mathrm{H})$, 3.48-3.35 (m, 2H), 2.68 (ddd, $J=13.7,8.3,5.6 \mathrm{~Hz}, 1 \mathrm{H}$ ), 2.37-2.30 (m, 1H); ${ }^{13} \mathrm{C}$ NMR $\left(100 \mathrm{MHz}, \mathrm{CDCl}_{3}\right) \delta 178.6,176.2$, $169.2,139.2,133.9,133.8,133.3,133.1,132.6,131.7,129.5$, 128.7, 128.5, 128.3, 127.1, 118.6, 117.2, 81.8, 37.0, 33.8; HRMS (Q-TOF, ESI) calcd for $\mathrm{C}_{24} \mathrm{H}_{18} \mathrm{BrN}_{2} \mathrm{O}_{2}+[\mathrm{M}+\mathrm{H}]+445.0546$, found 445.0544 .

1-Benzoyl-5-methyl-5'-phenyl-3',4'-dihydrospiro[indoline-3 ,2'-pyrrol]-2-one (3ma): yellow solid; $100.0 \mathrm{mg}$; $88 \%$ yield; mp $171-172{ }^{\circ} \mathrm{C} ;{ }^{1} \mathrm{H}$ NMR $\left(400 \mathrm{MHz}, \mathrm{CDCl}_{3}\right) \delta 7.93-7.91(\mathrm{~m}, 2 \mathrm{H})$, $7.86(\mathrm{~d}, J=8.3 \mathrm{~Hz}, 1 \mathrm{H}), 7.77-7.75(\mathrm{~m}, 2 \mathrm{H}), 7.56(\mathrm{t}, J=7.5 \mathrm{~Hz}$, $1 \mathrm{H}), 7.51-7.41(\mathrm{~m}, 53 \mathrm{H}), 7.23$ (dd, $J=8.3,1.0 \mathrm{~Hz}, 1 \mathrm{H}), 7.06$ (s, 1H), 3.43-3.39 (m, 2H), 2.67 (ddd, $J=13.7,8.1,5.8 \mathrm{~Hz}, 1 \mathrm{H}$ ), 2.38-2.30 (m, 4H); ${ }^{13} \mathrm{C}$ NMR $\left(100 \mathrm{MHz}, \mathrm{CDCl}_{3}\right) \delta 177.9,177.1$, $169.4,137.8,135.5,134.4,133.6,132.7,131.7,131.5,130.2$, 129.4, 128.6, 128.5, 128.3, 124.4, 115.4, 82.2, 37.0, 33.9, 21.3; HRMS (Q-TOF, ESI) calcd for $\mathrm{C}_{25} \mathrm{H}_{21} \mathrm{~N}_{2} \mathrm{O}_{2}{ }^{+}[\mathrm{M}+\mathrm{H}]+381.1598$, found 381.1582 .

1-Benzoyl-5-methoxy-5'-phenyl-3',4'-dihydrospiro[indoline -3,2'-pyrrol]-2-one (3na): yellow solid; $100.0 \mathrm{mg}$; $84 \%$ yield; mp 149-150 ${ }^{\circ} \mathrm{C}$; ${ }^{1} \mathrm{H}$ NMR $\left(400 \mathrm{MHz}, \mathrm{CDCl}_{3}\right) \delta 7.95-7.90(\mathrm{~m}, 3 \mathrm{H})$, 7.75-7.73 (m, 2H), 7.57-7.41 (m, 6H), $6.95(\mathrm{dd}, J=8.9,2.7 \mathrm{~Hz}$, $1 \mathrm{H}), 6.79$ (d, $J=2.7 \mathrm{~Hz}, 1 \mathrm{H}), 3.81(\mathrm{~s}, 3 \mathrm{H}), 3.42-3.38(\mathrm{~m}, 2 \mathrm{H})$, 2.68 (ddd, $J=13.5,8.4,5.2 \mathrm{~Hz}, 1 \mathrm{H}$ ), 2.37-2.29 (m, 1H); ${ }^{13} \mathrm{C} \mathrm{NMR}$ $\left(100 \mathrm{MHz}, \mathrm{CDCl}_{3}\right) \delta 178.2,176.9,169.3,157.9,134.5,133.5$, 
133.0, 132.6, 131.6, 129.3, 128.6, 128.5, 128.2, 116.8, 114.7, 109.9, 82.3, 55.9, 37.0, 34.0; HRMS (Q-TOF, ESI) calcd for $\mathrm{C}_{25} \mathrm{H}_{21} \mathrm{~N}_{2} \mathrm{O}_{3}+[\mathrm{M}+\mathrm{H}]+397.1547$, found 397.1539.

5'-(2-Chlorophenyl)-1-tosyl-3',4'-dihydrospiro[indoline-3,2' -pyrrol]-2-one (3ab): yellow solid; $65.0 \mathrm{mg}$; $48 \%$ yield; $\mathrm{mp}$ 134-135 ${ }^{\circ} \mathrm{C} ;{ }^{1} \mathrm{H}$ NMR $\left(400 \mathrm{MHz}, \mathrm{CDCl}_{3}\right) \delta 7.91(\mathrm{~d}, J=8.3 \mathrm{~Hz}, 2 \mathrm{H})$, $7.86(\mathrm{~d}, J=8.2 \mathrm{~Hz}, 1 \mathrm{H}), 7.52(\mathrm{dd}, J=7.7,1.6 \mathrm{~Hz}, 1 \mathrm{H}), 7.33-7.11$ (m, 8H), 3.45-3.29 (m, 2H), 2.53 (ddd, $J=13.5,8.8,4.8 \mathrm{~Hz}, 1 \mathrm{H}$ ), 2.32 (s, 3H), 2.21-2.13 (m, 1H); ${ }^{13} \mathrm{C}$ NMR (100 MHz, $\left.\mathrm{CDCl}_{3}\right) \delta$ 179.8, 175.0, 145.8, 139.0, 135.2, 133.9, 132.6, 131.35, 130.8, 130.4, 129.99, 129.96, 128.0, 127.0, 125.5, 124.2, 113.8, 81.1, 39.9, 35.1, 21.8; HRMS (Q-TOF, ESI) calcd for $\mathrm{C}_{24} \mathrm{H}_{20} \mathrm{ClN}_{2} \mathrm{O}_{3} \mathrm{~S}^{+}[\mathrm{M}$ $+\mathrm{H}]+451.0878$, found 451.0881 .

5'-(3-Chlorophenyl)-1-tosyl-3',4'-dihydrospiro[indoline-3,2' -pyrrol]-2-one (3ac): yellow solid; $95.4 \mathrm{mg} ; 71 \%$ yield; $\mathrm{mp}$ 148-149 ${ }^{\circ} \mathrm{C}$; ${ }^{1} \mathrm{H}$ NMR (400 MHz, $\left.\mathrm{CDCl}_{3}\right) \delta 8.00-7.94(\mathrm{~m}, 3 \mathrm{H})$, 7.84-7.83 (m, 1H), 7.67 (d, J = 7.7 Hz, 1H), 7.44-7.31 (m, 5H), $7.19(\mathrm{td}, J=7.5,0.8 \mathrm{~Hz}, 1 \mathrm{H}), 7.12(\mathrm{dd}, J=7.4,1.0 \mathrm{~Hz}, 1 \mathrm{H})$, 3.40-3.30 (m, 2H), 2.62 (ddd, $J=13.4,8.8,4.6 \mathrm{~Hz}, 1 \mathrm{H}$ ), 2.42 (s, $3 \mathrm{H}), 2.26-2.18(\mathrm{~m}, 1 \mathrm{H}) ;{ }^{13} \mathrm{C} \mathrm{NMR}\left(100 \mathrm{MHz}, \mathrm{CDCl}_{3}\right) \delta 177.5$, 175.2, 145.9, 139.1, 135.2, 135.1, 134.8, 131.5, 130.9, 130.1, 130.0, 129.9, 128.4, 128.1, 126.6, 125.6, 124.1, 113.9, 81.7, 36.6, 34.5, 21.8; HRMS (Q-TOF, ESI) calcd for $\mathrm{C}_{24} \mathrm{H}_{20} \mathrm{ClN}_{2} \mathrm{O}_{3} \mathrm{~S}^{+}[\mathrm{M}+$ $\mathrm{H}]^{+} 451.0878$, found 451.0868 .

5'-(4-Chlorophenyl)-1-tosyl-3',4'-dihydrospiro[indoline-3,2' -pyrrol]-2-one (3ad): yellow solid; $97.0 \mathrm{mg}$; $72 \%$ yield; mp 192-193 ${ }^{\circ} \mathrm{C}$; ${ }^{1} \mathrm{H}$ NMR $\left(400 \mathrm{MHz}, \mathrm{CDCl}_{3}\right) \delta 8.00-7.94(\mathrm{~m}, 3 \mathrm{H})$, 7.77-7.74 (m, 2H), 7.41-7.30 (m, 5H), 7.20-7.11 (m, 2H), 3.40-3.29 (m, 2H), 2.60 (ddd, $J=13.4,8.8,4.6 \mathrm{~Hz}, 1 \mathrm{H}$ ), 2.41 (s, $3 \mathrm{H}), 2.25-2.17(\mathrm{~m}, 1 \mathrm{H}) ;{ }^{13} \mathrm{C}$ NMR $\left(100 \mathrm{MHz}, \mathrm{CDCl}_{3}\right) \delta 177.6$, 175.3, 145.9, 139.0, 137.7, 135.2, 131.9, 131.0, 130.02, 129.97, 129.7, 128.9, 128.1, 125.5, 124.1, 113.9, 81.7, 36.6, 34.6, 21.8; HRMS (Q-TOF, ESI) calcd for $\mathrm{C}_{24} \mathrm{H}_{20} \mathrm{ClN}_{2} \mathrm{O}_{3} \mathrm{~S}^{+}[\mathrm{M}+\mathrm{H}]+451.0878$, found 451.0863 .

5'-(4-Fluorophenyl)-1-tosyl-3',4'-dihydrospiro[indoline-3,2' -pyrrol]-2-one (3ae): yellow solid; $101.2 \mathrm{mg}$; 78\% yield; $\mathrm{mp}$ 191-192 ${ }^{\circ} \mathrm{C} ;{ }^{1} \mathrm{H}$ NMR $\left(400 \mathrm{MHz}, \mathrm{CDCl}_{3}\right) \delta 7.99(\mathrm{~d}, J=8.3 \mathrm{~Hz}, 2 \mathrm{H})$, $7.95(\mathrm{~d}, J=8.3 \mathrm{~Hz}, 1 \mathrm{H}), 7.83(\mathrm{dd}, J=8.6,5.5 \mathrm{~Hz}, 2 \mathrm{H}), 7.38(\mathrm{t}, J=$ $7.2 \mathrm{~Hz}, 1 \mathrm{H}), 7.31(\mathrm{~d}, J=8.2 \mathrm{~Hz}, 2 \mathrm{H}), 7.18(\mathrm{t}, J=7.5 \mathrm{~Hz}, 1 \mathrm{H}), 7.13$ (d, $J=7.4 \mathrm{~Hz}, 1 \mathrm{H}), 7.06(\mathrm{t}, J=8.6 \mathrm{~Hz}, 2 \mathrm{H}), 3.38-3.27(\mathrm{~m}, 2 \mathrm{H})$, 2.60 (ddd, $J=13.4,8.6,4.8 \mathrm{~Hz}, 1 \mathrm{H}), 2.41$ (s, 3H), 2.24-2.17 (m, 1H); ${ }^{13} \mathrm{C}$ NMR $\left(100 \mathrm{MHz}, \mathrm{CDCl}_{3}\right) \delta 177.4,175.4,164.8(\mathrm{~d}, J=$ $252.3 \mathrm{~Hz}), 145.8,139.0,135.2,131.1,130.6(\mathrm{~d}, J=8.8 \mathrm{~Hz})$, 130.0, 129.7 (d, $J=3.1 \mathrm{~Hz}$ ), 128.1, 125.5, 124.1, 115.7 (d, $J=$ $21.8 \mathrm{~Hz}), 113.8,81.6,36.6,34.7,21.8 ;{ }^{19} \mathrm{~F}$ NMR (376 MHz, $\left.\mathrm{CDCl}_{3}\right) \delta-107.98$ HRMS (Q-TOF, ESI) calcd for $\mathrm{C}_{24} \mathrm{H}_{20} \mathrm{FN}_{2} \mathrm{O}_{3} \mathrm{~S}^{+}$ $[\mathrm{M}+\mathrm{H}]+435.1173$, found 435.1168 .

5'-(4-Bromophenyl)-1-tosyl-3',4'-dihydrospiro[indoline-3,2' -pyrrol]-2-one (3af): yellow solid; $113.2 \mathrm{mg} ; 76 \%$ yield; $\mathrm{mp}$ 196-197 ${ }^{\circ} \mathrm{C}$; ${ }^{1} \mathrm{H}$ NMR $\left(400 \mathrm{MHz}, \mathrm{CDCl}_{3}\right) \delta 8.00-7.94(\mathrm{~m}, 3 \mathrm{H})$, $7.68(\mathrm{~d}, J=8.6 \mathrm{~Hz}, 2 \mathrm{H}), 7.51(\mathrm{~d}, J=8.6 \mathrm{~Hz}, 2 \mathrm{H}), 7.38(\mathrm{td}, J=8.0$, $1.5 \mathrm{~Hz}, 1 \mathrm{H}), 7.31(\mathrm{~d}, J=8.1 \mathrm{~Hz}, 2 \mathrm{H}), 7.18(\mathrm{t}, J=7.1 \mathrm{~Hz}, 1 \mathrm{H}), 7.12$ (dd, $J=7.5,1.1 \mathrm{~Hz}, 1 \mathrm{H}$ ), 3.39-3.29 (m, 2H), 2.60 (ddd, $J=13.4$, 8.7, $4.7 \mathrm{~Hz}, 1 \mathrm{H}), 2.41$ (s, 3H), 2.25-2.17 (m, 1H); ${ }^{13} \mathrm{C}$ NMR (100 $\mathrm{MHz}_{\mathrm{CDCl}}$ ) $\delta 177.6,175.2,145.8,139.0,135.2,132.3,131.8$, 131.0, 130.00, 129.95, 129.9, 128.1, 126.2, 125.5, 124.1, 113.9, 81.7, 36.5, 34.6, 21.8; HRMS (Q-TOF, ESI) calcd for
$\mathrm{C}_{24} \mathrm{H}_{20} \mathrm{BrN}_{2} \mathrm{O}_{3} \mathrm{~S}^{+}[\mathrm{M}+\mathrm{H}]+495.0373$, found 495.0368.

5'-( -Tolyl)-1-tosyl-3',4'-dihydrospiro[indoline-3,2'-pyrrol]2-one (3ag): yellow solid; $104.2 \mathrm{mg}$; $81 \%$ yield; mp 146-147 ${ }^{\circ} \mathrm{C}$; ${ }^{1} \mathrm{H}$ NMR (400 MHz, $\mathrm{CDCl}_{3}$ ) $\delta 8.00$ (d, $\left.J=8.3 \mathrm{~Hz}, 2 \mathrm{H}\right), 7.94$ (d, $J$ $=8.2 \mathrm{~Hz}, 1 \mathrm{H}), 7.72(\mathrm{~d}, J=8.1 \mathrm{~Hz}, 2 \mathrm{H}), 7.39-7.35(\mathrm{~m}, 1 \mathrm{H}), 7.31(\mathrm{~d}$, $J=8.1 \mathrm{~Hz}, 2 \mathrm{H}), 7.20-7.11(\mathrm{~m}, 4 \mathrm{H}), 3.41-3.33(\mathrm{~m}, 2 \mathrm{H}), 2.63-2.56$ (m, 1H), 2.41 (s, 3H), $2.38(\mathrm{~s}, 3 \mathrm{H}), 2.23-2.15(\mathrm{~m}, 1 \mathrm{H}) ;{ }^{13} \mathrm{C} \mathrm{NMR}$ $\left(100 \mathrm{MHz}, \mathrm{CDCl}_{3}\right) \delta 178.6,175.6,145.8,142.1,139.0,135.3$, $131.4,130.7,130.0,129.9,129.3,128.5,128.1,125.5,124.2$, 113.8, 81.5, 36.5, 34.8, 21.8, 21.7; HRMS (Q-TOF, ESI) calcd for $\mathrm{C}_{25} \mathrm{H}_{23} \mathrm{~N}_{2} \mathrm{O}_{3} \mathrm{~S}^{+}[\mathrm{M}+\mathrm{H}]^{+} 431.1424$, found 431.1420 .

5'-(4-(tert-Butyl)phenyl)-1-tosyl-3',4'-dihydrospiro[indolin e-3,2'-pyrrol]-2-one (3ah): yellow solid; $110.0 \mathrm{mg}$; $78 \%$ yield; mp 182-183 ${ }^{\circ} \mathrm{C}$; ${ }^{1} \mathrm{H}$ NMR $\left(400 \mathrm{MHz}, \mathrm{CDCl}_{3}\right) \delta 8.00(\mathrm{~d}, J=8.3 \mathrm{~Hz}$, 2H), 7.95 (d, $J=8.2 \mathrm{~Hz}, 1 \mathrm{H}), 7.78$ (d, $J=8.4 \mathrm{~Hz}, 2 \mathrm{H}), 7.43-7.30$ (m, 5H), 7.19-7.11 (m, 2H), 3.40-3.32 (m, 2H), 2.60 (ddd, $J=$ 13.4, 8.7, $4.7 \mathrm{~Hz}, 1 \mathrm{H}), 2.41$ (s, 3H), 2.22-2.15 (m, 1H), 1.33 (s, 9H); ${ }^{13} \mathrm{C}$ NMR $\left(100 \mathrm{MHz}, \mathrm{CDCl}_{3}\right) \delta 178.5,175.5,155.2,145.8$, 139.0, 135.3, 131.4, 130.7, 129.9, 129.8, 128.3, 128.1, 125.5, $125.5,124.1,113.8,81.5,36.5,35.0,34.8,31.2,21.8$; HRMS (Q-TOF, ESI) calcd for $\mathrm{C}_{28} \mathrm{H}_{29} \mathrm{~N}_{2} \mathrm{O}_{3} \mathrm{~S}^{+}[\mathrm{M}+\mathrm{H}]^{+} 473.1893$, found 473.1872.

1-Methyl-3-methyleneindolin-2-one (4a): known product [37], ${ }^{1} \mathrm{H}$ NMR (400 MHz, $\left.\mathrm{CDCl}_{3}\right) \delta 7.47(\mathrm{~d}, J=7.4 \mathrm{~Hz}, 1 \mathrm{H}), 7.30$ (dd, $J=7.8,1.0 \mathrm{~Hz}, 1 \mathrm{H}), 7.03(\mathrm{td}, J=7.6,0.9 \mathrm{~Hz}, 1 \mathrm{H}), 6.81$ (d, $J=$ $7.8 \mathrm{~Hz}, 1 \mathrm{H}), 6.40(\mathrm{~s}, 1 \mathrm{H}), 6.10(\mathrm{~s}, 1 \mathrm{H}), 3.24(\mathrm{~s}, 3 \mathrm{H})$; HRMS (Q-TOF, ESI) calcd for $\mathrm{C}_{10} \mathrm{H}_{10} \mathrm{NO}^{+}[\mathrm{M}+\mathrm{H}]^{+} 160.0757$, found 160.0790 .

\section{Results and discussion}

Our investigation started with the optimization of the reaction conditions, using the easily prepared 3-diazo-1-methylindolin-2-one (1a) and (1-azidovinyl)benzene (2a) reactants as model substrates. The initial experiments were performed by heating $1 \mathrm{a}$ and $2 \mathrm{a}$ ( 3 equiv.) at $60{ }^{\circ} \mathrm{C}$ in toluene for $10 \mathrm{~h}$, in the presence of $\mathrm{AgSbF}_{6}(10 \mathrm{~mol} \%)$ or $\mathrm{PPh}_{3} \mathrm{AuCl} / \mathrm{AgSbF}_{6}(10 \mathrm{~mol} \%, \mathrm{Au} / \mathrm{Ag}=1: 1)$. However, the desired product 3aa was not detected under these conditions (Table 1, entries 1 and 2). On the other hand, the dirhodium carboxylates showed high efficiency for this same transformation, giving the desired product 3aa in moderate yields (Table 1, entries 3-5). The structure of 3aa was unambiguously confirmed by ${ }^{1} \mathrm{H}$ and ${ }^{13} \mathrm{C}$ NMR, along with HRMS and single-crystal X-ray diffraction measurements. This encouraging result prompted us to examine the effect of various reaction parameters, such as solvent and temperature, and the results are summarized in Table 1 . Screening of different solvents showed that the best results were achieved with DCE (entries 6-10). Considering the decomposition of $\mathbf{2 a}$, the yield of $\mathbf{3 a a}$ increased to $81 \%$ when 7 equiv. of $2 \mathbf{a}$ was employed in the reaction (entries 11-13). We also investigated different temperatures, with no improvement in the reaction outcome (entries 14 and 15). Moreover, decreasing the amount of catalyst resulted in a lower yield (entry 16). Hence, the optimized reaction conditions were determined to be $\mathbf{1 a}(0.1 \mathrm{mmol}), \mathbf{2 a}(0.7$ mmol), $\mathrm{Rh}_{2}$ (TFA) 4 (2.5 mol\%), and DCE (1 mL), heated at $60{ }^{\circ} \mathrm{C}$ 
Table 1

Optimization of the reaction conditions.
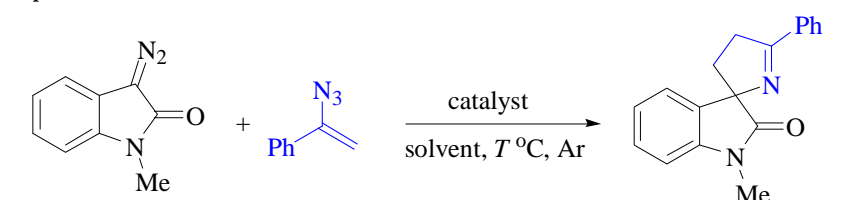

\begin{tabular}{|c|c|c|c|c|}
\hline 1a & $2 a$ & & & Заa \\
\hline Entry & Catalyst & Solvent & $T\left({ }^{\circ} \mathrm{C}\right)$ & Yield a (\%) \\
\hline 1 & $\mathrm{AgSbF}_{6}$ & toluene & 60 & 0 \\
\hline 2 & $\mathrm{PPh}_{3} \mathrm{AuCl} / \mathrm{AgSbF}_{6}$ & toluene & 60 & 0 \\
\hline 3 & $\mathrm{Rh}_{2}(\mathrm{OAc})_{4}$ & toluene & 60 & 59 \\
\hline 4 & $\mathrm{Rh}_{2}(\mathrm{Oct})_{4}$ & toluene & 60 & 57 \\
\hline 5 & $\mathrm{Rh}_{2}(\mathrm{TFA})_{4}$ & toluene & 60 & 60 \\
\hline 6 & $\mathrm{Rh}_{2}(\mathrm{TFA})_{4}$ & $\mathrm{PhCl}$ & 60 & 62 \\
\hline 7 & $\mathrm{Rh}_{2}(\mathrm{TFA})_{4}$ & $\mathrm{PhCF}_{3}$ & 60 & 54 \\
\hline 8 & $\mathrm{Rh}_{2}(\mathrm{TFA})_{4}$ & DCE & 60 & 67 \\
\hline 9 & $\mathrm{Rh}_{2}(\mathrm{TFA})_{4}$ & DCM & 60 & 67 \\
\hline 10 & $\mathrm{Rh}_{2}(\mathrm{TFA})_{4}$ & 1,4-dioxane & 60 & 42 \\
\hline $11^{b}$ & $\mathrm{Rh}_{2}(\mathrm{TFA})_{4}$ & DCE & 60 & 73 \\
\hline $12^{c}$ & $\mathrm{Rh}_{2}(\mathrm{TFA})_{4}$ & DCE & 60 & $81(79)^{e}$ \\
\hline $13^{d}$ & $\mathrm{Rh}_{2}(\mathrm{TFA})_{4}$ & DCE & 60 & 74 \\
\hline $14 \mathrm{c,f}$ & $\mathrm{Rh}_{2}(\mathrm{TFA})_{4}$ & DCE & 40 & 70 \\
\hline $15^{c}$ & $\mathrm{Rh}_{2}(\mathrm{TFA})_{4}$ & DCE & 80 & 77 \\
\hline $16^{\mathrm{c}, \mathrm{g}}$ & $\mathrm{Rh}_{2}(\mathrm{TFA})_{4}$ & DCE & 60 & 76 \\
\hline
\end{tabular}

Reaction conditions: 1a (0.1 mmol), 2a (3 equiv.), catalyst (10 mol\%), dirhodium carboxylates (2.5 mol\%), solvent $(1 \mathrm{~mL}), \mathrm{Ar}, 10 \mathrm{~h}$.

a Yields were measured by high-performance liquid chromatography (HPLC). ${ }^{\mathrm{b}} \mathbf{2 a}$ (5 equiv.). ${ }^{\mathrm{c}} \mathbf{2 a}$ (7 equiv.). ${ }^{\mathrm{d}} \mathbf{2 a}$ (9 equiv.). ${ }^{\mathrm{e}}$ Isolated yields. ${ }^{\mathrm{f}}$ 24 h. ${ }^{g} \mathrm{Rh}_{2}$ (TFA) 4 (1 mol\%).

under argon atmosphere for $10 \mathrm{~h}$.

Having determined the optimal reaction conditions, we explored the generality of the present approach. The results are summarized in Scheme 3.

The $N$-protecting groups of substrate 1 were first investigated. To our delight, various protecting groups including alkyl, benzyl, phenyl, benzoyl, and sulfonyl groups provided good results (3aa-3fa). In particular, the yields of 3da and 3ea reached up to $90 \%$, while the methylsulfonyl group led to a decrease in the product yield (3fa, 74\%). Notably, unprotected 3-diazooxindole $(\mathbf{1 g})$ reacted with $\mathbf{2 a}$ as well, generating the target product 3ga in $80 \%$ yield. Next, the substituents on the phenyl ring of $\mathbf{1}$ were examined. Substituents with different electronic effects on the phenyl ring of the 1-benzoyl-3-diazooxindole (1d) compound were well tolerated (3ha-3na). With a halogen $(\mathrm{Cl})$ group at the C5-, C6- or C7-position of the phenyl ring, the annulation resulted in the corresponding spirocyclic products in moderate to good yields (3ha-3ja). The steric effect of 1-benzoyl-7-chloro-3-diazooxindole (1i) caused a small decrease in the yield (75\% vs. $85 \%)$. The electronic effect of substituents at the C5-position had little influence on the yields (3ja-3na, 82\%-89\%). A similar phenomenon was observed when various substituents were introduced in the phenyl group of the vinyl azide 2 , yielding the products in moderate to good yields (3ab-3ah, 48\%-81\%).

Control experiments were conducted to investigate the reaction mechanism (Scheme 4). After stirring a mixture of $\mathbf{1 a}$, 2a, and $\mathrm{Rh}_{2}(\mathrm{TFA})_{4}$ in DCE at $60^{\circ} \mathrm{C}$ for $1 \mathrm{~h}$, an unexpected prod-
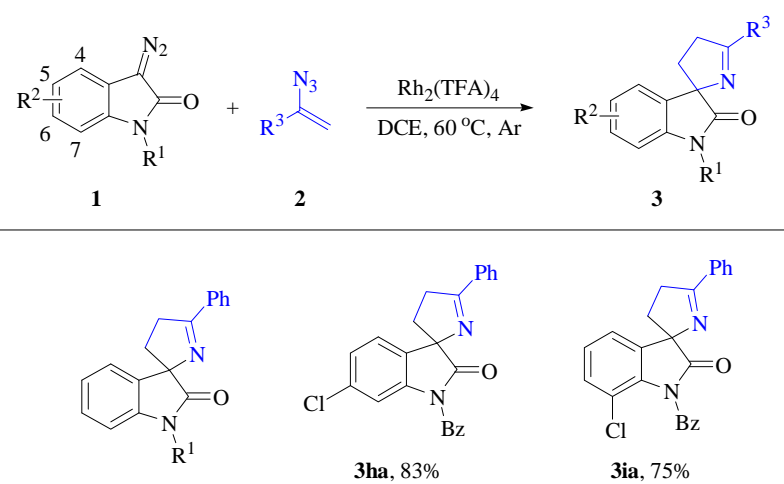

$\mathrm{R}^{1}=\mathrm{Me}, \mathbf{3 a a}, 79 \%$

$\mathrm{R}^{1}=\mathrm{Bn}, \mathbf{3 b a}, 88 \%$

$\mathrm{R}^{1}=\mathrm{Ph}, \mathbf{3 c a}, 89 \%$

$\mathrm{R}^{1}=\mathrm{Bz}, \mathbf{3 d a}, 91 \%$

$\mathrm{R}^{1}=\mathrm{Ts}$, 3ea, $90 \%$

$\mathrm{R}^{1}=\mathrm{Ms}, 3 \mathbf{3}$ a, $74 \%$

$\mathrm{R}^{1}=\mathrm{H}$, 3ga, $79 \%$
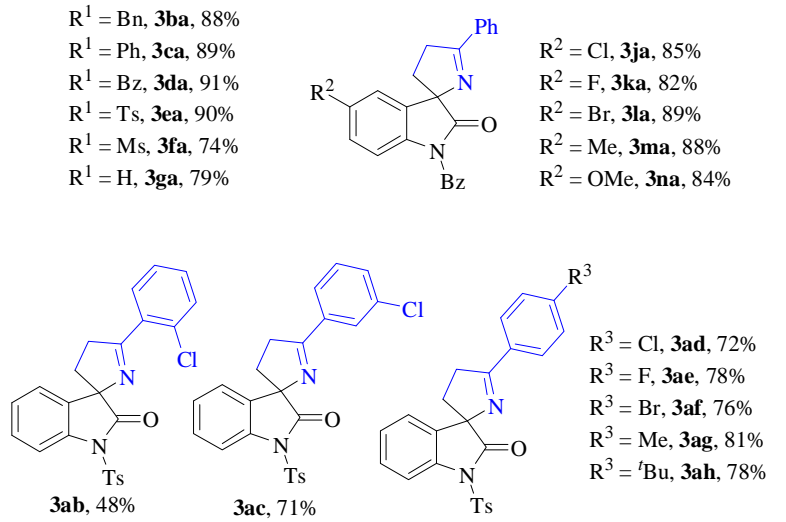

Scheme 3. Substrate scope. Reaction conditions: 1 (0.3 mmol), 2 (2.1 $\mathrm{mmol}), \mathrm{Rh}_{2}(\mathrm{TFA})_{4}(2.5 \mathrm{~mol} \%)$, DCE $(3 \mathrm{~mL})$, heated at $60^{\circ} \mathrm{C}$ under argon atmosphere for $10 \mathrm{~h}$. Isolated yields are shown.

uct (4a) was isolated in 52\% yield, in addition to the desired product 3aa (34\% yield). Since a $79 \%$ yield of product 3aa was obtained after prolonging the reaction time to $10 \mathrm{~h}$ (Table 1 , entry 12), it is reasonable to conclude that compound $\mathbf{4 a}$ was the key intermediate of the process. In addition, when the reaction was carried out at room temperature (rt) for $10 \mathrm{~h}$, compound $\mathbf{4 a}$ was observed as well. The reaction of $4 \mathbf{a}$ with $2 \mathbf{a}$ was also conducted under standard reaction conditions, affording the desired product 3aa in 67\% yield (Scheme 4).

On the basis of these results [34], a plausible mechanism was proposed, using the reaction of the 3-diazooxindole 1a and vinyl azide 2a as an example (Scheme 5). The Rh(II)-catalyzed denitrogenation of $\mathbf{1 a}$ generates the electrophilic rhodium carbenoid A. Next, the nucleophilic addition of $\mathbf{2 a}$ to the carbenoid
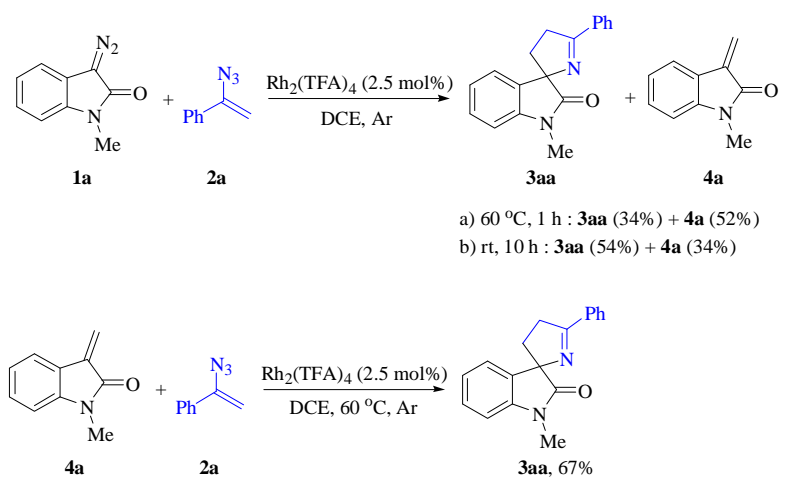

Scheme 4. Control experiments. 


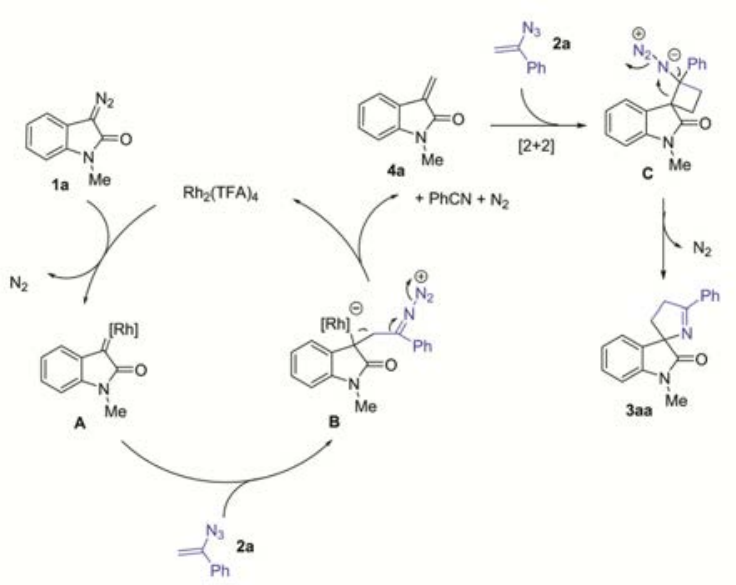

Scheme 5. Proposed reaction mechanism.

A gives the intermediate $\mathbf{B}$, followed by the formation of the key intermediate $4 \mathbf{a}$ with loss of $\mathrm{N}_{2}$ and phenylacetonitrile. It should be noted that although the intermediate $4 \mathbf{a}$ can be isolated, it is unstable. Finally, compound 4 a reacts with another molecule of $\mathbf{2 a}$ to yield the product 3aa through a [2+2] cycloaddition/ring expansion sequence.

\section{Conclusions}

Potentially bioactive spiropyrrolidine oxindoles were synthesized by the $\mathrm{Rh}(\mathrm{II})$-catalyzed $[1+1+3]$ annulation of 3-diazooxindoles and vinyl azides through an olefination/cyclization sequence. This transformation is highly efficient and tolerates various substituents. Owing to its mild conditions, broad scope, and high efficiency, this approach is likely to find application in the synthesis of a wide range of spirocyclic compounds.

\section{References}

[1] R. Rios, Chem. Soc. Rev., 2012, 41, 1060-1074

[2] C. Marti, E. M. Carreira, Eur. J. Org. Chem., 2003, 2209-2219.

[3] C. V. Galliford, K. A. Scheidt, Angew. Chem. Int. Ed., 2007, 46, 8748-8758.

[4] J. H. Lu, J. Q. Tan, S. S. K. Durairajan, L. F. Liu, Z. H. Zhang, L. Ma, H.
M. Shen, H. Y. E. Chan, M. Li, Autophagy, 2012, 8, 98-108.

[5] G. G. Cheng, Y. L. Zhao, Y. Zhang, P. K. Lunga, D. B. Hu, Y. Li, J. Gu, C. W. Song, W. B. Sun, Y. P. Liu, X. D. Luo, Tetrahedron, 2014, 70, 8723-8729.

[6] H. J. Cong, Q. Zhao, S. W. Zhang, J. J. Wei, W. Q. Wang, L. J. Xuan, Phytochemistry, 2014, 100, 76-85.

[7] Y. Arun, K. Saranraj, C. Balachandran, P. T. Perumal, Eur. J. Med. Chem., 2014, 74, 50-64.

[8] A. V. Velikorodov, V. A. Ionova, O.V. Degtyarev, L. T. Sukhenko, Pharm. Chem. J., 2013, 46, 715-719.

[9] K. Ding, Z. Han, Z. Wang, Chem. Asian J., 2009, 4, 32-41.

[10] N. R. Ball-Jones, J. J. Badillo, A. K. Franz, Org. Biomol. Chem., 2012, 10, 5165-5181.

[11] G. S. Singh, Z. Y. Desta, Chem. Rev., 2012, 112, 6104-6155.

[12] L. Hong, R. Wang, Adv. Synth. Catal., 2013, 355, 1023-1052.

[13] F. Shi, Z. L. Tao, S. W. Luo, S. J. Tu, L. Z. Gong, Chem. Eur. J., 2012, 18, 6885-6894.

[14] R. Rajesh, R. Raghunathan, Eur. J. Org. Chem., 2013, 2013, 2597-2607.

[15] K. Suman, L. Srinu, S. Thennarasu, Org. Lett., 2014, 16, 3732-3735.

[16] H. B. Yang, Y. Wei, M. Shi, Tetrahedron, 2013, 69, 4088-4097.

[17] Y. M. Cao, F. F. Shen, F. T. Zhang, R. Wang, Chem. Eur. J., 2013, 19, 1184-1188.

[18] F. Tan, L. Q. Lu, Q. Q. Yang, W. Guo, Q. Bian, J. R. Chen, W. J. Xiao, Chem. Eur. J., 2014, 20, 3415-3420.

[19] M. S. Poslusney, B. J. Melancon, P. R. Gentry, D. J. Sheffler, T. M. Bridges, T. J. Utley, J. S. Daniels, C. M. Niswender, P. J. Conn, C. W. Lindsley, M. R. Wood, Bioorg. Med. Chem. Lett., 2013, 23, 1860-1864.

[20] D. Chen, M. H. Xu, Chem. Commun., 2013, 49, 1327-1329.

[21] B. Zhang, P. Feng, L. H. Sun, Y. Cui, S. Ye, N. Jiao, Chem. Eur. J., 2012, 18, 9198-9203.

[22] J. L. Meloche, B. L. Ashfeld, Angew. Chem. Int. Ed., 2017, 56, 6604-6608.

[23] B. V. S. Reddy, E. P. Reddy, B. Sridhar, Y. J. Rao, RSC Adv., 2016, 6, 50497-50499.

[24] S. Muthusamy, C. Gunanathan, M. Nethaji, J. Org. Chem., 2004, 69, 5631-5637.

[25] B. Hu, S. G. DiMagno, Org. Biomol. Chem., 2015, 13, 3844-3855.

[26] J. Fu, G. Zanoni, E. A. Anderson, X. Bi, Chem. Soc. Rev., 2017, 46, 7208-7228.

[27] P. Gu, Y. Su, X. P. Wu, J. Sun, W. Liu, P. Xue, R. Li, Org. Lett., 2012, 14, 2246-2249.

[28] E. López, L. A. López, Angew. Chem. Int. Ed., 2017, 56, 5121-5124.

[29] R. Yi, X. Li, B. Wan, Adv. Synth. Catal., 2018, 360, 875-880.

\section{Graphical Abstract}

Chin. J. Catal., 2019, 40: 177-183 doi: 10.1016/S1872-2067(18)63200-0

Synthesis of spiropyrrolidine oxindoles through Rh(II)-catalyzed olefination/cyclization of diazooxindoles and vinyl azides

Ruxia Yi, Leilei Qian, Boshun Wan*

Dalian Institute of Chemical Physics, Chinese Academy of Sciences;

University of Chinese Academy of Sciences

An efficient $[1+1+3]$ annulation process of 3-diazooxindoles and vinyl azides has been developed using dirhodium carboxylate as catalyst, providing access to potential bioactive spiropyrrolidine oxindoles in moderate to good yields and with a broad substrate scope.

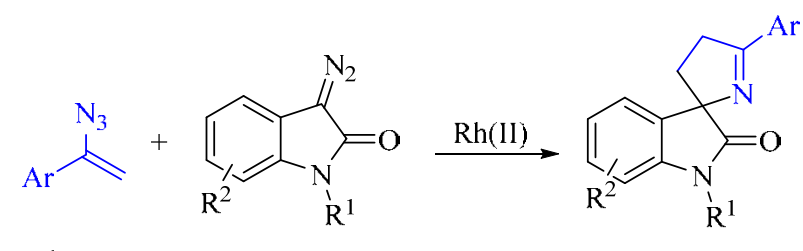

$\mathrm{R}^{1}=\mathrm{H}$, alkyl, aryl, benzoyl, sulfonyl

$\mathrm{R}^{2}=$ halogen, alkyl, alkoxyl 
[30] Y. Hu, R. Yi, X. Yu, X. Xin, C. Wang, B. Wan, Chem. Commun., 2015, 51, 15398-15401.

[31] Y. Zhao, Y. Hu, H. Wang, X. Li, B. Wan, J. Org. Chem., 2016, 81, 4412-4420.

[32] T. Li, F. Xu, X. Li, C. Wang, B. Wan, Angew. Chem. Int. Ed., 2016, 55, 2861-2865.

[33] C. Wang, D. Wang, H. Yan, H. Wang, B. Pan, X. Xin, X. Li, F. Wu, B. Wan, Angew. Chem. Int. Ed., 2014, 53, 11940-11943.
[34] V. Kanchupalli, S. Katukojvala, Angew. Chem. Int. Ed., 2018, 57, 5433-5437.

[35] B. Alcaide, P. Almendros, C. Aragoncillo, R. Callejo, M. P. Ruiz, M. R. Torres, Eur. J. Org. Chem., 2012, 2012, 2359-2366.

[36] D. Cantillo, B. Gutmann, C. Oliver Kappe, Org. Biomol. Chem., 2016, $14,853-857$.

[37] M. A. Loreto, A. Migliorini, P. A. Tardella, A. Gambacorta, Eur. J. Org. Chem., 2007, 2365-2371.

\title{
Rh(II)催化3-重氮吲哚酮与烯基叠氮的烯化/环化反应合成螺吡咯啉吲哚酮
}

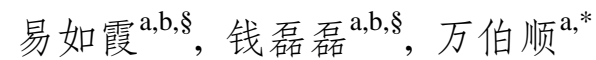 \\ $\mathrm{a}$ 中国科学院大连化学物理研究所, 辽宁大连116023 \\ b 中国科学院大学, 北京 100049
}

\begin{abstract}
摘要: 螺吡咯啉吲哚酮化合物含有两种重要氮杂环, 由于其独特的结构骨架和存在于天然产物而被广泛关注. 吲喍酮的 3 位与其它环状化合物以螺环形式结合的结构特点是该结构具有潜在药物活性和合成价值的基础, 例如抗癌和抗菌活性, 以 及在合成新配体和有机催化剂上的应用. 目前, 尽管合成螺吲哚酮的策略已有1,3-偶极环加成、亲核加成及还原环化等, 但 是发展简单高效的构建螺吲哚酮化合物的方法仍具有很大的吸引力.

烯基叠氮同时含有叠氮和烯基两个单元, 被广泛应用于构建氮杂环. 另一方面, 重氮化合物被广泛用作偶联环化合成 的底物. 基于在叠氮化学和杂环合成方面的工作, 我们设想利用3-重氮吲哚-2-酮和烯基氮的环化反应构建螺吲哚酮化合 物. 文献中有关烯基叠氮和重氮化合物反应的报道较少, 主要涉及铑催化的环丙烷化和铜催化的环戊烯合成, 在这些反应 中烯基叠氮作为二元合成子参与反应, 而其它类型的反应鲜有报道, 因此我们设想利用烯基叠氮作为三元合成子来参与反 应成环. 在我们开展工作的同时, Katukojvala小组率先发表了铑催化的重氮烯和烯基叠氮的环化反应构建1-吡咯啉.

本文报道了3-重氮吲哚酮和烯基叠氮在铑催化下发生 $[1+1+3]$ 环化, 构建一系列螺吡咯啉吲哚酮化合物. 研究从反应 条件优化开始, 通过对催化剂、原料比、溶剂和温度等参数的篮选, 确定了最佳反应条件为 $\mathbf{1 a} / \mathbf{2 a}(1 / 7), \mathrm{Rh}_{2}(\mathrm{TFA})_{4}(2.5$ $\mathrm{mol} \%), 1,2$ 二二氯乙烷 $(0.1 \mathrm{~mol} / \mathrm{L}), 6{ }^{\circ} \mathrm{C}$. 在标准条件下完成了 21 个不同基团取代的螺吡咯啉吲哚酮化合物的合成, 最高收 率可达 $91 \%$, 证实了该反应的普适性. 当重氮底物的 $\mathrm{N}$ 原子上不含取代基或取代基为甲基、芐基、苯基、苯甲酰基和磺酰 基时, 反应均可以顺利发生, 其中苯甲酰基和对甲苯磺酰基取代的底物的反应可取得 $90 \%$ 上的收率. 对于重氮和烯基叠 氮底物的苯环上含有卤素、甲基和甲氧基等取代基时, 反应同样可以顺利进行, 以中等收率得到对应产物, 电子效应对反应 效果影响不大, 而存在位阻效应时反应收率略有降低. 当降低反应温度或缩短反应时间, 可以从反应体系中同时分离得到 螺吡咯啉吲哚酮和重氮底物3位乙烯基化的产物. 进一步实验表明, 3-烯基吲哚酮可以在标准条件下与烯基叠氮反应, 以中 等收率得到模板产物. 该对照实验表明3-烯基吲哚酮是反应过程中的关键中间体. 该反应条件温和, 简单高效, 底物适用 范围广, 为构建具有潜在生物活性的螺吲哚酮骨架提供了新的选择.
\end{abstract}

关键词：铑催化剂; 烯基叠氮; 重氮化合物; 螺吡咯啉㖿哚酮; 乙烯基化; [1+1+3]环化

收稿日期: 2018-11-02. 接受日期: 2018-11-14. 出版日期: 2019-02-05.

*通讯联系人. 电话: (0411)84379260; 传真: (0411)84379223; 电子信箱: bswan@dicp.ac.cn

易如霞和钱否磊对本工作贡献相同.

基金来源：国家自然科学基金(21572225).

本文的电子版全文由Elsevier出版社在ScienceDirect上出版(http://www.sciencedirect.com/science/journal/18722067). 